\title{
Re-modeling ELISA kits embedded in an automated system suitable for on- line detection of algal toxins in seawater
}

\author{
Konstantinos Petropoulos ${ }^{\mathrm{a}}$, Sergio F. Bodini ${ }^{\mathrm{b}, * *}$, Laura Fabiani ${ }^{\mathrm{a}}$, Laura Micheli ${ }^{\mathrm{a}}$, \\ Alessandro Porchetta ${ }^{a}$, Silvia Piermarini ${ }^{a}$, Giulia Volpe ${ }^{a, *}$, Fabio Massimo Pasquazzi $^{\mathrm{b}}$, \\ Luca Sanfilippo $^{\mathrm{b}}$, Pompeo Moscetta ${ }^{\mathrm{b}}$, Salvatore Chiavarini ${ }^{\mathrm{c}}$, Giuseppe Palleschi ${ }^{\mathrm{a}}$ \\ ${ }^{\text {a }}$ Department of Chemical Science and Technologies, University of Rome "Tor Vergata", Via della Ricerca Scientifica, Rome, Italy \\ ${ }^{\mathrm{b}}$ Systea SpA, via Fratta Rotonda Vado Largo, 2/A, 03012 Anagni, (FR), Italy \\ ${ }^{\mathrm{c}}$ ENEA CR Casaccia, SSPT-PROTER Division, via Anguillarese 301, 00123 Rome, Italy
}

\section{A R T I C L E I N F O}

\section{Keywords:}

ELIMC assays

ASMAT

Marine algal toxins

Seawater

On-line monitoring

\begin{abstract}
A B S T R A C T
The increasing demand by citizens and environmental organization for the protection, preservation, and possible restoration of the marine environment has made seawater protection one of the urgent priorities of the European Union. In this contest, extensive monitoring and surveillance are required to correctly assess the current status of marine environment, paying attention not only to traditional chemical parameters, but also to the so-called "emerging pollutants". Among these, marine biotoxins represent a serious hazard, because they can accumulate in fish and mussels and enter the food chain. The detection of marine toxins released by algae has mostly been directed on the analysis of fish/shellfish homogenate rather than seawater samples. To fill this gap, in this work, we propose the re-modeling of ELISA kits to set-up non-automated direct competitive ELIMC (Enzyme-Linked Immuno-Magnetic Colorimetric) assays for the detection of Domoic Acid (DA), Okadaic Acid (OA) and Saxitoxin (STX) in seawater.

For each toxin, linear working range, sensitivity and detection limit $(0.03,0.05,0.01 \mathrm{ng} / \mathrm{mL}$ for DA, OA and STX, respectively) of the ELIMC assays were calculated. After evaluation of seawater matrix effect and recovery study, ELIMC assays were integrated within a novel automated networked system (ASMAT - Analytical System for Marine Algal Toxins) based on the micro Loop Flow Reactor ( $\mu$ LFR) technology, suitable for on-line monitoring of marine toxins. Once established the best operational conditions, ASMAT was calibrated towards DA, OA and STX verifying its capability to detect sub-ppb levels of the target toxins. Analysis of DA, OA and STX in real samples of marine water, sampled in Alonnisos (Greece) were carried out in laboratory using ELIMC assays, ASMAT and ELISA Kits, for a comparative evaluation. Finally, on-line suitability of ASMAT as an early warning alarm system was demonstrated by a field installation within a floating platform in the port of La Spezia (Italy).
\end{abstract}

\section{Introduction}

Harmful Algal Blooms (HABs) are a common phenomenon in coastal and fresh water ecosystems caused by colonies of algae that grow out of control often producing toxic effects on people, fish, shellfish, marine mammals, and birds [1]. HABs occur naturally due to algae transported via storms, sea currents and river flows. Their growth is stimulated by light and warmer water temperature in the summer. However, the main reason for the increased frequency of HABs in the recent decades [2,3] is constituted by anthropogenic activity, including excessive nutrient loading from fertilizers or sewage waste, food web alterations, introduced species, water flow modifications and climate changes. The term HABs is related to non-toxic and toxic algae blooms. Due to biomass accumulation, non-toxic HABs damage the aquatic ecosystems by suffocating fish, blocking sunlight from reaching bottom-dwelling plants, depleting the oxygen in the water after their death, when an increased activity of decomposing microorganisms occurs. Toxic HABs involve microalgae (in marine water) and cyanobacteria (in fresh and brackish waters) that produce toxic secondary metabolites able to alter cellular processes of other organisms from plankton to humans through the food web [2,3]. Among the thousands of microalgal species known in nature, about 300 are involved in harmful events and more than 100

\footnotetext{
* Corresponding author at: Department of Chemical Science and Technologies "Tor Vergata" University, Via della Ricerca Scientifica, Rome, Italy.

** Corresponding author at: SYSTEA SpA, Via Fratta Rotonda Vado Largo, 2/A, 03012 Anagni, (FR), Italy.

E-mail addresses: sergio.bodini@systea.it (S.F. Bodini), giulia.volpe@uniroma2.it (G. Volpe).
} 
(essentially diatoms and dinoflagellates) produce persistent natural toxins that can cause intoxication or even death in humans and animals. It is well known that marine biotoxins can accumulate in tissues of marine organisms, particularly filter-feeding bivalves (i.e. mussels filter approximately $20 \mathrm{~L}$ water/h). Outbreaks of intoxication in humans due to marine biotoxins are caused by the ingestion of contaminated shellfish and can have a wide range of symptoms due to the specific toxic compound [4].

On the basis of their poisoning symptoms, marine biotoxins are classified as toxins causing paralytic shellfish poisoning (PSP), amnesic shellfish poisoning (ASP), diarrheic shellfish poisoning (DSP), neurotoxic shellfish poisoning (NSP), and ciguatera fish poisoning (CFP) [5]. Focusing on the first three groups, PSP is caused by saxitoxin (STX) and its analogues, ASP is caused by domoic acid (DA), and DSP is associated with a group of polyether toxins including okadaic acid (OA), the dinophysistoxins, pectenotoxins, and yessotoxins [4].

To protect consumer's health, the Commission of the European Community has set different safe intake levels for the presence of these toxins in bivalve molluscs (EC Regulation No 853/2004) and has established monitoring and sampling plans in the production areas of live bivalve molluscs (EC Regulation No 854/2004). To check compliance with the limits laid down, Mouse and Rat Bioassays (MBA, RBA) have been for many years considered as the reference monitoring methods for phycotoxins. The major drawbacks of these bioassays are a limited sensitivity, high cost, variability and lack of specificity. In addition, interferences can lead to false positive results. For these reasons, and to avoid or minimize the use of living animals, the European Commission established the replacement of these methods [6]. Following these recommendations, several methods have been validated as alternative to the MBA and RBA to detect marine biotoxins in edible part of molluscs, as reported by Botana [7]. In the case of PSP, the total content of STXgroup toxins must be officially detected by MBA (EC Regulation No 2074/2005) or by AOAC HPLC fluorimetric method, so-called Lawrence method (EC Regulation No 1664/2006). In the case of ASP, the total content of DA group toxins can be detected by using either an HPLC-UV or an ELISA screening method (EC Regulation No 1244/2007). Finally, LC-MS/MS was approved to be the reference method (EU Regulation 15/2011) for the detection of DSP toxins, even if the MBA could be used as screening method for new and unknown toxins.

No legislation is currently available for the testing of marine biotoxins in algal or seawater samples and, consequently, no official methods have been approved by the European Commission. Nevertheless, a number of methods are reported for toxin analysis in algal samples and less frequently in seawater, mostly of them based on HPLC coupled with fluorescence or UV detection [8-13]. In addition, LC-MS/MS [14-18] has been employed for PSP, DSP and ASP toxins. All these methods, called chemical methods, are time-consuming (i.e. sample derivatization, sample clean-up, sample pre-concentration step) and can be used only in equipped laboratories by highly qualified staff.

A plethora of immunological assays have been developed as biochemical tests for the screening of marine phycotoxins in shellfish. Among them, ELISA tests are commercially available as simple and rapid kits (e.g. Biosense Laboratories, Abraxis, Mercury Science, Bioo Scientific, Europroxima). As reported in the literature [19-26], some of these kits and other ELISA based methods, not commercially available, have been employed for the analysis of biotoxins in field and/or cultured algae and seawater samples. Great attention should be paid to two interesting immunological assays, based on SPR [21] and planar wave guide [27], developed by McNamee et al. for a multi-toxin analysis in cultured algae and seawater samples.

Of note, the analysis of seawater by immunological tests is often carried out by measuring, in the collected samples, the intracellular content of biotoxins released after lysis of algal cells.

We would like to underline that all the mentioned chemical and biochemical methods are based on manual handling and thus are not suitable for real-time monitoring of the marine environment.
Our work was carried out in the frame of the European project SMS (Sensing toxicants research in Marine waters make Sense using biosensors) aiming to realize a novel automated networked system to be used for real-time in situ monitoring of marine water chemical and ecological status in coastal areas. Given our long experience in the development of immuno-magnetic tests [28-33], and focusing the attention on the measurement of algal toxins, here we describe the set-up of non-automated direct competitive ELIMC (Enzyme-Linked ImmunoMagnetic Colorimetric) assays as the basis to construct an automated prototype suitable for on-line continuous monitoring of DA, OA and STX in seawater. We decided to detect free algal toxins directly in seawater for two main reasons: (i) no algal cell lysis is required and (ii) these compounds have been detected in seawater even in the absence of the producing algae [16].

The proposed ELIMC assays were designed by combining the advantages of magnetic beads (MBs) with the high performing and stable reagent solutions supplied by commercial ELISA kits. In particular, the 96-wells micro-plates included in the kits were replaced by MBs used as support of the immunological complex. Magnetic beads in fact can be easily transferred and integrated in an automated flow system suitable to be located in buoys across marine areas of Europe. To achieve this goal, we developed ASMAT (Analytical System for Marine Algal Toxins), a fully automated on-line analyzer based on the micro Loop Flow Reactor ( $\mu$ LFR) technology [34], that enables unattended analysis of Domoic Acid, Okadaic Acid and Saxitoxin. This novel analytical device was at first assessed in laboratory and then installed on a floating platform for on-line monitoring of the three algal toxins.

\section{Materials and methods}

\subsection{Reagents}

\subsubsection{Direct competitive ELIMC assays}

Domoic Acid ELISA kit was from EuroProxima (Arnhem, The Netherlands); Okadaic acid and Satitoxin ELISA-kits were purchased from Abraxis (Warminster, PA, USA). Reagents and materials included in the kits are reported in Supplementary material (S1). Saxitoxin standards in seawater $(0,0.02,0.05,0.1,0.2,0.4 \mathrm{ng} / \mathrm{mL})$ were also purchased from Abraxis and are stable at $2-8^{\circ} \mathrm{C}$. Goat Anti-Rabbit IgG magnetic beads (supplied as a suspension containing $3.65 \times 10^{10}$ beads $/ \mathrm{mL}$ ) were purchased from New England Biolabs (Ipswich, MA, UK). Certified calibration solutions of OA, DA and STX, purchased from the National Research Council (Ottawa, Canada), were used for the evaluation of seawater matrix effect and for recovery studies. For crossreactivity study DTX1, DTX2 and GTX2\&3 were provided from the National Research Council, whereas palytoxin (PITX) and brevetoxin (BTX) were from Wako Chemicals (Neuss, Germany) and Santa Cruz Biotechnology (Dallas, USA), respectively. Non-fat dry milk blotting grade was purchased from Bio-Rad (Hercules, CA, USA). All the other chemicals of analytical grade were purchased from Merck (Darmstadt, Germany).

\subsubsection{ASMAT}

The same reagents used for the non-automated ELIMC assays were employed to test the automated prototype.

\subsection{Materials and apparatus}

\subsubsection{Direct competitive ELIMC assays}

96-well polystyrene microtitre plates were purchased from Nunc (Roskilde, Denmark). A rotary shaker and a magnetic rack/particle concentrator (MPC) were from Dynal Biotech (Lake Success, NY). A Model iMark ${ }^{\mathrm{TM}}$ micro-plate reader (Bio-Rad, Hercules, CA, USA) was used for spectrophotometric detection.

A schematic representation of the ELIMC assay principle is reported in Fig. 1. As shown, the interaction between Anti-Rabbit IgG, 


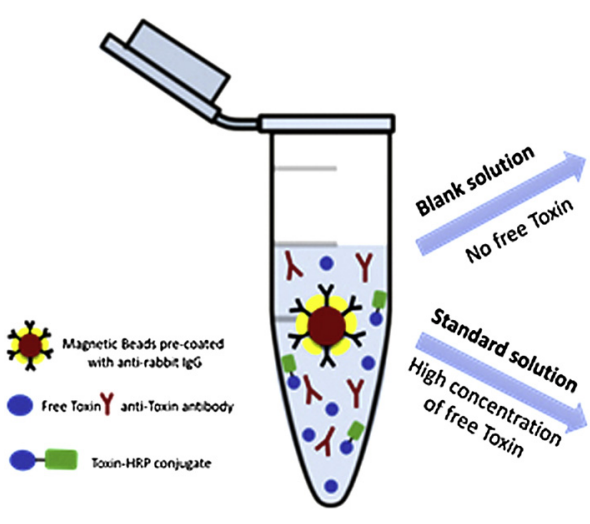

Single step procedure-Incubation $(30-60) \mathrm{min}$
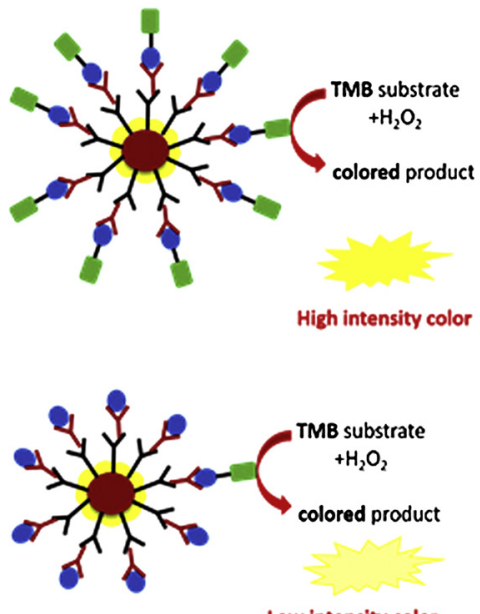

Fig. 1. Schematic representation of the ELIMC assay for detection of DA, OA and STX.

immobilized on the MBs surface, and Rabbit IgG (specific for the target toxin), as well as the competition between toxin and toxin-HRP conjugate, towards the specific antibody, take place in a single and short step procedure, carried out into a micro-tube. The non-bound reagents are then discarded by capturing the MBs (that support the formed immunological complex) with a magnet. MBs are then dissolved in a suitable HRP substrate solution. After a short incubation time, a stop solution is added and the activity of the captured enzyme is spectrophotometrically detected by measuring the rate of the color production. In the presence of toxin, competition occurs and consequently the rate of color production decreases proportionally to the concentration of the toxin.

\subsubsection{ASMAT}

ASMAT is a multi-parameter monitoring system housed in a portable plastic case (46 (L) x $36(\mathrm{~W}) \times 18(\mathrm{H}) \mathrm{cm}$ ) equipped with a LCD colour touch screen control pad (Fig. 2A). The storage, hydraulic, incubation and measuring components were designed, built and assembled by Systea laboratories (Anagni, Italy).

The base plate accommodates the liquid handling system, refrigerated and unrefrigerated reagent positions, the electrovalves and a colorimetric flow cell (Fig. 2B). The instrument was conceived to automatically perform sequential chemical analyses of seawater samples.

The sampling system was developed to allow direct intake of seawater followed by $0.1 \mu \mathrm{m}$ filtration and transfer into ASMAT. The groundwork of the competition assay is the incubation and detection system that consists of $5 \times 10 \times 10 \mathrm{~mm}$ Suprasil Quartz flow cell with $5 \mathrm{~mm}$ light path in which the immunomagnetic reactions take place at controlled temperature $\left(25^{\circ} \mathrm{C}\right)$. An optical interference filter is used to attenuate the blue LED emission and measure the Absorbance at $450 \mathrm{~nm}$. The cuvette is connected to a mechanical arm provided with a Neodymium magnet that allows for trapping the magnetic beads when requested by the procedure. The hydraulic circuit includes two peristaltic pumps: one for washing, sampling and emptying the system, with a higher flow rate, $2.4 \mathrm{~mL} / \mathrm{min}$ in slow mode, $4.8 \mathrm{~mL} / \mathrm{min}$ in fast mode, whereas the other, working at $0.3 \mathrm{~mL} / \mathrm{min}$, is for dosing microlitre volumes of reagents.

Because most of the reagents required for the method need to be refrigerated at $4{ }^{\circ} \mathrm{C}$, a refrigerated compartment, composed of an isolated aluminium block cooled by a Peltier element, was designed and developed to contain two compartments for $10 \mathrm{~mL}$ and eight for $4 \mathrm{~mL}$ vials. The washing solutions are stored at room temperature. To inject any reagent or washing solution into the $\mu \mathrm{LFR}$, the specific valve connected with the reagent vial or the washing bottle is opened for a defined time; simultaneously, one of the pumps is moving and the loop is opened to ensure that the exact volume is introduced inside the system.
The analyzer is managed via dedicated software which provides overall control of analytical operations and data acquisition functions by a GSM/GPRS device.

\subsection{Procedures}

\subsubsection{Direct competitive ELIMC assays}

The 96-wells micro-plates provided with the commercial kits for DA, OA and STX, were replaced by magnetic particles (MBs) pre-coated with Goat Anti-Rabbit IgG. In a preliminary phase, MBs were washed and blocked (to avoid nonspecific adsorptions) as reported below.

2.3.1.1. Preliminary washing-blocking procedure of MBs. After resuspension of the Goat Anti-Rabbit IgG magnetic beads, $500 \mu \mathrm{L}$ were pipetted into a $2 \mathrm{~mL}$ Eppendorf tube and washed twice in $1 \mathrm{~mL}$ of PBS pH $7.4+0.1 \%$ dry milk. The supernatant was discarded and the MBs were resuspended in $1 \mathrm{~mL}$ of PBS pH $7.4+3 \%$ dry milk (as blocking agent) and incubated for $30 \mathrm{~min}$ at room temperature (RT) slow tilt rotation (using the rotary shaker). The blocked MBs were washed twice in $1 \mathrm{~mL}$ of PBS pH $7.4+0.1 \%$ dry milk. Finally, the particles were resuspended in $500 \mu \mathrm{L}$ of PBS $+0.1 \%$ dry milk $+0.02 \%$ $\mathrm{NaN}_{3}$. The pre-coated and blocked MBs are stable for 3 months if stored at $4{ }^{\circ} \mathrm{C}$. Between each washing step, the Eppendorf tube was placed in the MPC for $2 \mathrm{~min}$ and the supernatant was discarded.

The pre-coated and blocked MBs were then used as a solid phase to set up a competitive immunoassay for each toxin.

\subsubsection{Competitive immunoassay procedure for domoic acid detection.}

1 Shake and transfer $10 \mu \mathrm{L}$ of the pre-coated and blocked beads suspension (stored at $4{ }^{\circ} \mathrm{C}$ ) into a $2 \mathrm{~mL}$ Eppendorf tube (in a number required for the calibrants and/or samples to analyze);

2 wash 3 times with $1 \mathrm{~mL}$ of Rinsing buffer $1 \mathrm{X}$, every time shaking and discarding the supernatant by placing the tubes MPC;

3 add in each Eppendorf tube:

a) $150 \mu \mathrm{L}$ of ready to use standard solutions of DA $(0,0.05,0.1,0.2$, $0.5,1,2 \mathrm{ng} / \mathrm{mL}$ ) or seawater samples;

b) $75 \mu \mathrm{L}$ of DA-HRP solution (diluted as described in 2.1 section);

c) $75 \mu \mathrm{L}$ of rabbit anti-DA antibody (dissolved as reported in 2.1 section);

4 incubate for $30 \mathrm{~min}$ at RT with slow tilt rotation;

5 wash 3 times with $1 \mathrm{~mL}$ of Rinsing buffer $1 \mathrm{X}$, every time shaking and discarding the supernatant.

2.3.1.3. Competitive immunoassay procedure for okadaic acid detection. 

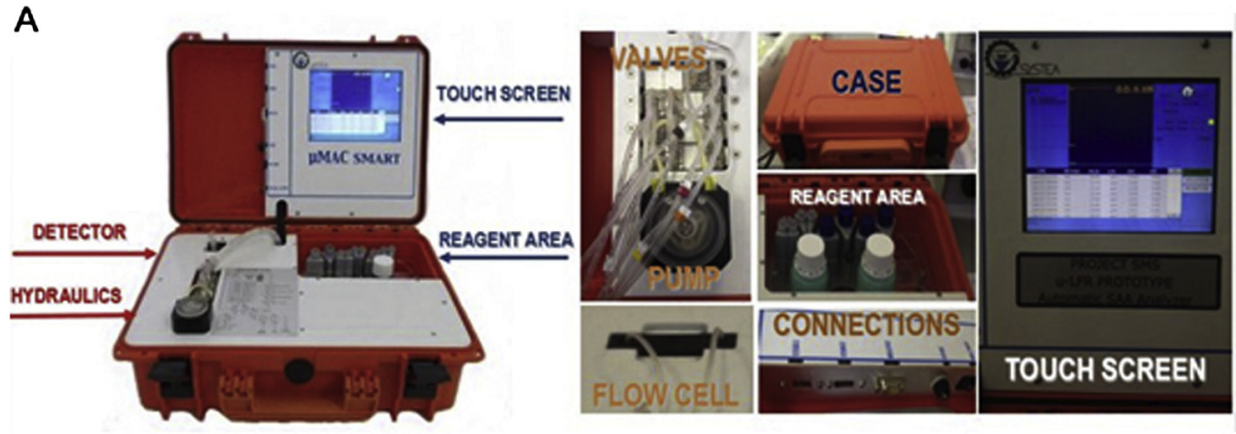

Fig. 2. A) ASMAT housed in a portable plastic case (46 (L) x $36(\mathrm{~W}) \times 18(\mathrm{H}) \mathrm{cm}$ ) equipped with a LCD colour touch screen control pad; B) Scheme of the ASMAT organization: (1) sampling device including microfilter and sampling bag; (2) refrigerated positions for reagents and storage area for washing solutions; (3) flow injection system; including pumps, tubes and valves; (4) flow cell inclusive of optical device, mechanical arm for magnet positioning and heating system; (5) control and data processing system and communication module.

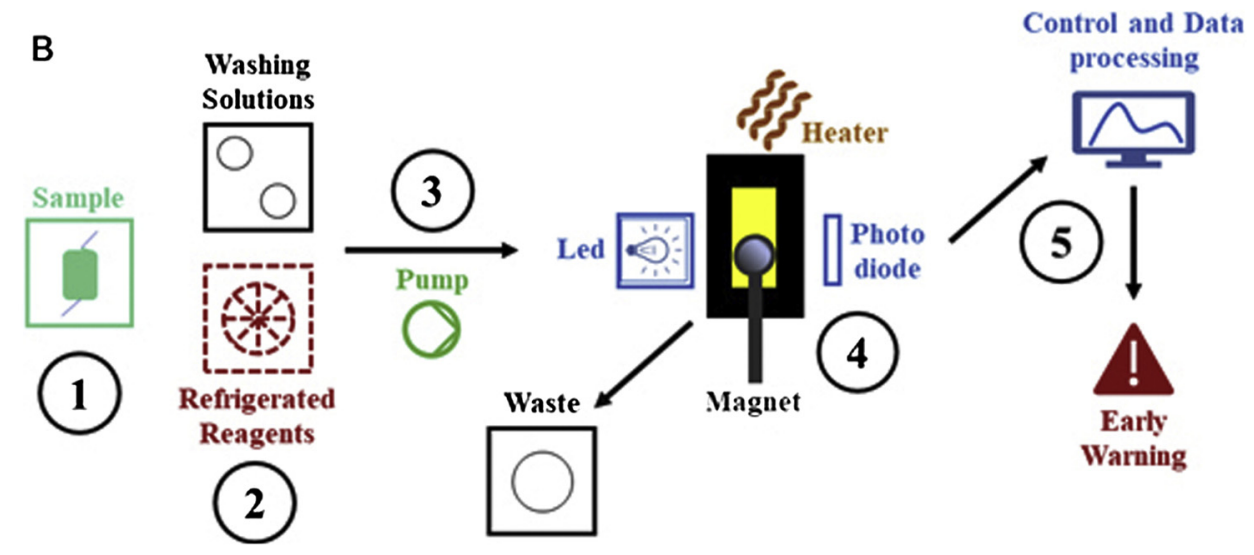

1 Shake and transfer $10 \mu \mathrm{L}$ of the coated and blocked beads suspension (stored at $4{ }^{\circ} \mathrm{C}$ ) into a $2 \mathrm{~mL}$ Eppendorf tube (in a number required for the calibrants and/or samples to analyze);

2 wash 3 times with $1 \mathrm{~mL}$ of Wash Solution $1 \mathrm{X}$, every time shaking and discarding the supernatant by placing the tubes MPC;

3 add in each Eppendorf tube:

a $200 \mu \mathrm{L}$ of ready to use standard solutions of OA $(0,0.1,0.2,0.5,1$, $2,5 \mathrm{ng} / \mathrm{mL}$ ) or seawater samples;

b $100 \mu \mathrm{L}$ of OA-HRP solution;

c $100 \mu \mathrm{L}$ of rabbit anti-OA antibody;

4 incubate for $60 \mathrm{~min}$ with slow tilt rotation at RT.

5 wash 3 times with $1 \mathrm{~mL}$ of Wash solution $1 \mathrm{X}$, every time shaking and discarding the supernatant.

\subsubsection{Competitive immunoassay procedure for saxitoxin detection.}

1 Shake and transfer $10 \mu \mathrm{L}$ of the coated and blocked beads suspension (stored at $4{ }^{\circ} \mathrm{C}$ ) into a $2 \mathrm{~mL}$ Eppendorf tube (in a number required for the calibrants and/or samples to analyze);

2 wash 3 times with $1 \mathrm{~mL}$ of Wash solution $1 \mathrm{X}$, every time shaking and discarding the supernatant by placing the tubes MPC;

3 add in each Eppendorf tube:

a $100 \mu \mathrm{L}$ of ready to use standard solutions of STX $(0,0.02,0.05$,

$0.1,0.2,0.4 \mathrm{ng} / \mathrm{mL}$ ) or seawater samples;

b $100 \mu \mathrm{L}$ of STX-HRP solution;

c $100 \mu \mathrm{L}$ of rabbit anti-STX antibody;

4 incubate for $30 \mathrm{~min}$ with slow tilt rotation at RT.

5 wash 3 times with $1 \mathrm{~mL}$ of Wash solution $1 \mathrm{X}$, every time shaking and discarding the supernatant.

2.3.1.5. Colorimetric measurements. The MBs contained in each Eppendorf tube were resuspended in a suitable volume of the TMB-kit solution: $300 \mu \mathrm{L}$ for DA and OA assays, $200 \mu \mathrm{L}$ for STX assay. After an incubation time of $15 \mathrm{~min}$ (for DA), $20 \mathrm{~min}$ (for OA) and $30 \mathrm{~min}$ (for
STX), at RT with slow tilt rotation, a volume of the $\mathrm{H}_{2} \mathrm{SO}_{4}$-kit solution ( $300 \mu \mathrm{L}$ for DA and $200 \mu \mathrm{L}$ for OA and STX) was added to block the enzymatic reaction and mixed well. Finally, $100 \mu \mathrm{L}$ of each suspension (three replicates for each toxin) were transferred into separate wells of a microtiter plate and the absorbance was read at $450 \mathrm{~nm}$.

\subsubsection{ASMAT}

The manual DA, OA and STX assays were properly adapted to the prototype and the automated procedures are described in Supplementary material (S2).

To achieve early warning protection, the system was programmed to automatically generate an alarm immediately after detecting the presence of a toxin.

\subsubsection{Seawater sample collection in Alonnisos}

The sampling in Alonnisos was done on May $11^{\text {th }}$ and $12^{\text {th }}, 2017$. It was done on 2 locations, one onshore (coastal) and one offshore (pelagic) in order to capture possible water quality variations, depending on location. On day 1 , the coastal surface sample of $20 \mathrm{~L}$ was collected from Station1 (S1) near the port of Alonnisos. The depth of S1 was $40 \mathrm{~cm}$ and its distance from the coast was $2 \mathrm{~m}$. On day 2, $20 \mathrm{~L}$ of pelagic surface sample were collected from Station 2 (S2) in Alonnisos National Marine Park. The depth of S2 was $90 \mathrm{~m}$ and its distance from the coast was $760 \mathrm{~m}$.

Water samples were collected by filling $5 \mathrm{~L}$ pitchers $10-20 \mathrm{~cm}$ below the water surface from the two sampling stations. They were filtered within $2 \mathrm{~h}$ after sampling on both days 1 and 2 . One liter of each sample was filtered through $0.45 \mu \mathrm{m}$ PVDF filters. The filtrates were kept frozen during the shipment to our laboratory where the analysis of DA, OA and STX was carried out using ELIMC assays, ASMAT and ELISA kits. 


\section{Results and discussion}

The first part of the present study describes the set-up of manual direct competitive ELIMC (Enzyme-Linked Immuno-Magnetic Colorimetric) assays to detect DA, OA and STX in seawater. These assays were designed by combining the high performing reagent solutions supplied by the commercial ELISA kits (Abraxis and Europroxima) with magnetic beads, used as support of the immunological complex. These kits have been selected, among those commercially available, because the primary antibodies (rabbit IgG, specific for the target toxins) are in solution and therefore it is possible to immobilize them on an external appropriate support. In particular, the 96-wells micro-plates pre-coated with Anti-Rabbit IgG, able to bind the Fc region of rabbit IgG, included in the kits, were replaced by MBs pre-coated with Goat Anti-Rabbit IgG.

The second part of this work was aimed to design and assemble a novel analytical system suitable for on-line monitoring of DA, OA and STX in seawater, without the need of operator intervention. Thanks to the use of MBs, it was possible to transfer and integrate the manual assays in an automated prototype, ASMAT, designed as a multi-parameter flow analyzer.

\subsection{Direct competitive ELIMC assays}

The ELIMC approach adopted in this work allows to combine the selectivity of the antibody, the convenience of a separation step (through the use of magnetic beads) and the straightforwardness of colorimetric detection. All these features match well with the challenge to develop an efficient system for in situ continuous monitoring of algal toxins in seawater.

The use of magnetic particles, instead of the 96-wells micro-plates (included in the kits), has resulted in the optimization of their volume, to be added into each Eppendorf tube, and the selection of the best blocking agent allows for avoiding unspecific adsorptions on their surface. $10 \mu \mathrm{L}$ of MBs (pre-coated with Anti-Rabbit IgG) and 3\% dry milk were chosen and used to construct calibration curves for DA, OA and STX (following the steps reported in 2.3.1.2, 2.3.1.3, 2.3.1.4 and 2.3.1.5 sections).

In Fig. $3 \mathrm{~A}-\mathrm{C}$, a comparison between competition curves (for DA, OA and STX) carried out using ELIMC assays and the corresponding ELISA kits are reported.

The curves were constructed by plotting the $\% \mathrm{~B} / \mathrm{B}_{0}$ (by dividing the OD values of each standard for the mean OD value of the zero standard and multiplying by 100) on the linear Y-axis versus the corresponding toxin concentration on the logarithmic X-axis. The data of OA and STX were fitted using a linear regression, while for DA they were fitted using a nonlinear four-parameter logistic equation plot:

$\mathrm{f}(\mathrm{x})=\mathrm{y}_{\mathrm{o}}+\mathrm{a} / 1+\left(\mathrm{x} / \mathrm{x}_{0}\right)^{\mathrm{b}}$

in which $a$ and $y_{0}$ are the asymptotic maximum and minimum values, respectively, $b$ is the slope, and $x_{0}$ is the $x$ value at the inflection
Table 1

Comparison between detection limit (LOD), sensitivity $\left(50 \% \mathrm{~B} / \mathrm{B}_{0}\right)$ and working range (WR) obtained by ELISA kits and ELIMC assays, for each target toxin.

\begin{tabular}{|c|c|c|c|c|c|c|}
\hline & \multicolumn{2}{|l|}{ DA } & \multicolumn{2}{|l|}{$\mathrm{OA}$} & \multicolumn{2}{|l|}{ STX } \\
\hline & ELISA & ELIMC & ELISA & ELIMC & ELISA & ELIMC \\
\hline LOD (ng/mL) & 0.02 & 0.03 & 0.04 & 0.05 & 0.01 & 0.01 \\
\hline $50 \% \mathrm{~B} / \mathrm{B}_{0}(\mathrm{ng} / \mathrm{mL})$ & 0.36 & 0.42 & 0.50 & 0.83 & 0.06 & 0.07 \\
\hline $\mathrm{WR}(\mathrm{ng} / \mathrm{mL})$ & \multicolumn{2}{|c|}{$0.1-2.0$} & \multicolumn{2}{|c|}{$0.1-5.0$} & \multicolumn{2}{|c|}{$0.02-0.4$} \\
\hline
\end{tabular}

point.

For each toxin, linear working range, sensitivity $\left(50 \% \mathrm{~B} / \mathrm{B}_{0}\right)$ and detection limit (LOD), defined as the concentration corresponding to the $f(x)$ value obtained by subtracting three standard deviation of zero point from the mean of zero standard measurements, were calculated for ELISA and ELIMC assays and the results are reported in Table 1.

All the results obtained indicate that the performances of ELIMC assays are comparable to those of the ELISA kits; the advantage of ELIMC assays is the possibility to be integrated into an automated flowcolorimetric system, as later demonstrated.

As the ELISA kits for DA and OA, whose reagents have been used to set up the ELIMC assays, are suitable for quantitative and/or qualitative detection of the toxins in shellfish samples (as reported in the data sheets) and the analysis of seawater samples is not specified, we decided to test the possible matrix effect of seawater on the performances of the ELIMC assays. A seawater sample, collected from the natural reserve of Orbetello (Italy), was used as blank (endogenous content of the target toxins below the detection limits of the ELIMC assays). The experimental tests were performed analyzing in parallel toxin standard solutions included in the kits, and the same concentrations prepared in seawater. As shown in Fig. 4 A and B, seawater do not affect the response of the assays. The same experiments were carried out using ELISA kits with similar outcomes (data not shown).

On the other hand, the ELISA kit for STX is suitable for the detection of the toxin in shellfish and water (freshwater and seawater) samples. As seawater affects the response of the kit, Abraxis recommends the use of suitable saxitoxin standards in seawater, commercially available outside the ELISA kit. These solutions and also STX standard solutions, prepared in Orbetello seawater, were analyzed by ELIMC assay to evaluate the matrix effect. The experimental results were compared with those obtained using STX standard solutions included in the kit (Fig. 4C). A reduction of the linearity range $(0.05-0.4 \mathrm{ng} / \mathrm{mL}$ in seawater instead of $0.02-0.4 \mathrm{ng} / \mathrm{mL}$ in buffer) was observed. Importantly, the same matrix effect was observed using the ELISA kit (data not shown).

Cross-reactivity of each ELIMC assay was evaluated towards toxins belonging to the same class of the target and to other classes. Among the toxins reported in the data sheets of the ELISA kits, we tested the most readily commercially available. The results obtained (see Table S1
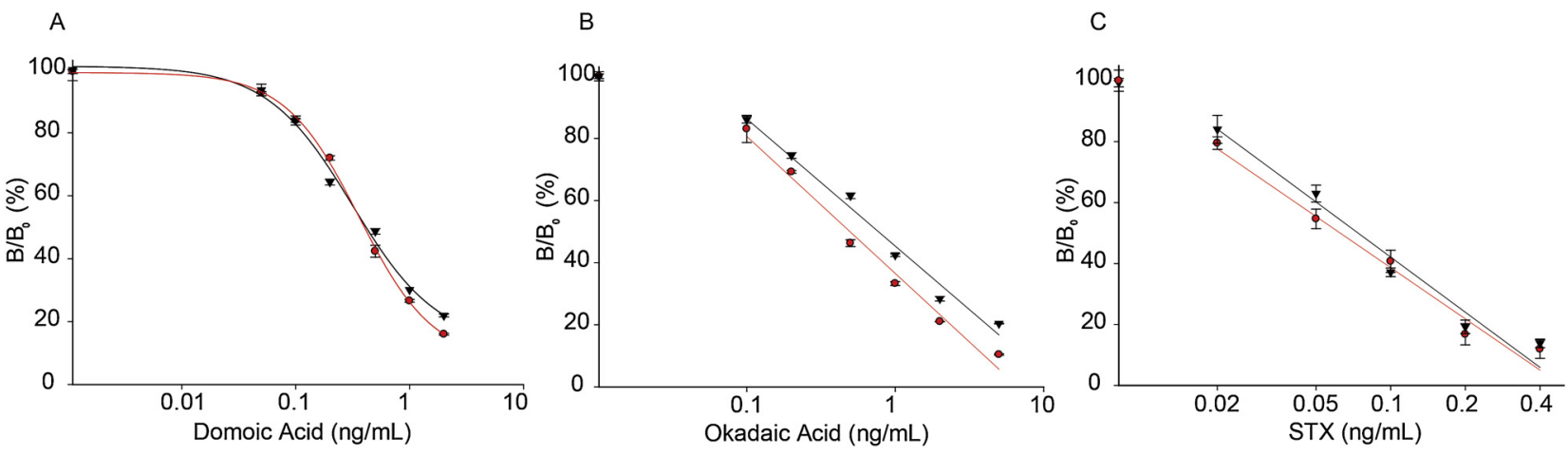

Fig. 3. Comparison between calibration curves obtained using ELISA kits (•) and ELIMC assays ( $)$ ); DA (A), OA (B), STX (C). 

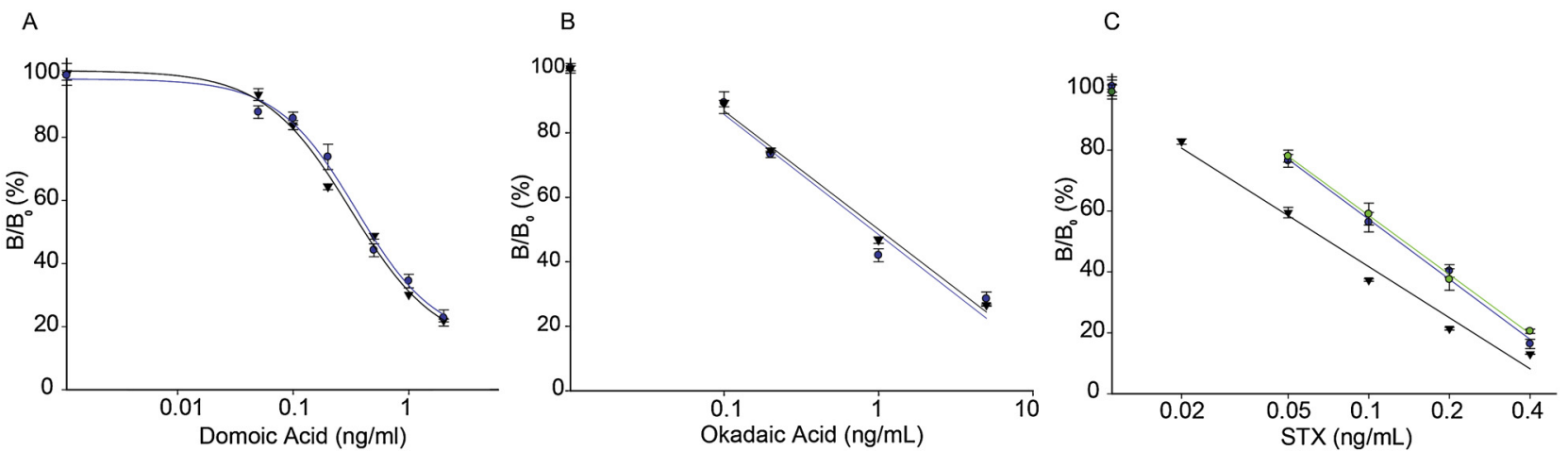

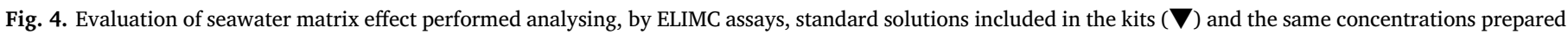
in Orbetello seawater (•); DA (A), OA (B) and STX (C). For STX, standard solutions in seawater (commercially available) were also analysed (॰).

in supplementary materials) were similar to those of the ELISA Kits.

Precision and recovery of the ELIMC assays were assessed by performing a blind analysis of different aliquots of the blank seawater sample, independently fortified by ENEA with fixed amount of the target toxins. We tested in triplicate three concentration levels of DA, $\mathrm{OA}$ and STX, repeating the analysis in three different days $(\mathrm{n}=9$ for each concentration, 3 replicates $\mathrm{x} 3$ days). The results are reported in Table 2.

For STX, given the presence of a matrix effect, the quantification was carried out by constructing the calibration curve with STX standards in seawater (Abraxis).

The same spiked samples were analyzed in parallel by ELISA kits obtaining similar results.

\subsection{ASMAT}

The manual ELIMC assays were integrated within a fully automated system for the detection of DA, OA and STX. This is the result of a strict and mutual collaboration between University of Tor Vergata and Systea which has a long experience in developing, manufacturing and testing automated analyzers for measuring chemical parameters in water.

The idea was to realize at first a prototype able to recognize and quantify one algal toxin: domoic acid. Subsequently, by varying some parameters set for DA, the analysis was extended to the other harmful toxins: STX and OA.

The main variables that were studied during a preliminary testing phase were: amount of magnetic beads, flow cell (designed to alternatively act also as a reactor, incubator and magnetic chamber), number of washings, reagent container positions, reagent volumes and analytical times. In particular, the system architecture, including reagent dilution and circuit washings, was optimized to solve problems associated with carryover and affinity binding of the antibodies to plastic materials.

Table 2

Precision (RSD\%) and recovery (R\%) of ELIMC assays obtained analyzing seawater samples spiked with three concentration levels of each target toxin.

\begin{tabular}{lllll}
\hline Toxin & $\begin{array}{l}\text { Spiked level } \\
\mathrm{ng} / \mathrm{mL}\end{array}$ & $\begin{array}{l}\text { Measured concentration (mean } \pm \mathrm{sd} \text { ) } \\
\mathrm{ng} / \mathrm{mL}\end{array}$ & $\mathrm{RSD} \%$ & $\mathrm{R} \%$ \\
\hline \multirow{2}{*}{ DA } & 0.14 & $0.16 \pm 0.02$ & 12.5 & 114 \\
& 0.42 & $0.37 \pm 0.03$ & 8.1 & 88 \\
& 1.00 & $0.93 \pm 0.08$ & 8.6 & 93 \\
OA & 0.30 & $0.26 \pm 0.03$ & 11.5 & 87 \\
& 1.48 & $1.30 \pm 0.11$ & 8.5 & 88 \\
& 3.05 & $2.80 \pm 0.30$ & 10.7 & 92 \\
STX & 0.02 & n.d. & - & - \\
& 0.10 & $0.10 \pm 0.02$ & 20 & 100 \\
& 0.40 & $0.46 \pm 0.04$ & 8.7 & 115 \\
\hline
\end{tabular}

* each value is the mean of 9 measurements ( 3 replicates $\mathrm{x} 3$ days).

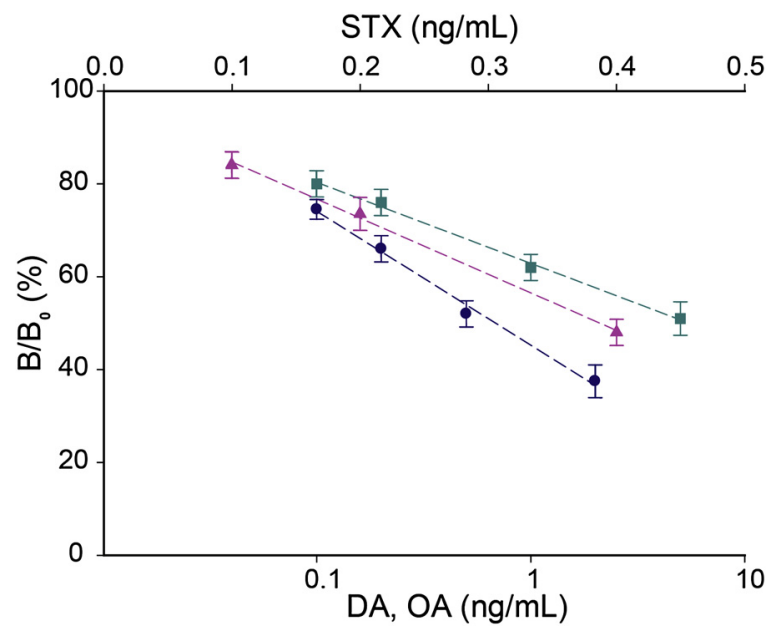

Fig. 5. Calibration plots obtained analysing, by ASMAT, standard solutions of DA (•), OA ( $\square)$ and STX (A).

Once established the best operational conditions, the measurement module was calibrated towards DA, OA and STX (Fig. 5). For STX giving the presence of a matrix effect (see Fig. 4C), the prototype was calibrated using seawater standards from Abraxis.

The analytical parameters (working ranges, $50 \% \mathrm{~B} / \mathrm{B}_{0}$ and LODs), less performing than that obtained using the non-automated ELIMC assays, are reported in Table 3.

It was observed that the average $\mathrm{RSD}$ of the $\mathrm{B} / \mathrm{B}_{0} \%$ signal ( $\left.\mathrm{RSD}_{\text {resp }}\right)$ for OA measurement (Fig. 5) was $5 \%$ but, given the poor slope of the calibration curve, an average $\mathrm{RSD}$ of the concentration $\left(\mathrm{RSD}_{\text {dose }}\right)$ equal to $30 \%$ was calculated. For this reason, it was not possible to distinguish between close concentrations (i.e. 0.1 and $0.2 \mathrm{ng} / \mathrm{mL}$ ) of OA. However, since the $0.1 \mathrm{ng} / \mathrm{mL}$ standard generated a signal decrease of about $20 \%$ compared to the blank measurements, a semi-quantitative analysis of OA could be performed. As for DA and STX, although the calculated average $\mathrm{RSD}_{\text {dose }}$ was 20 and $15 \%$, respectively, it was possible to

Table 3

Detection limit (LOD), sensitivity $\left(50 \% \mathrm{~B} / \mathrm{B}_{0}\right)$ and working range (WR) obtained by ASMAT, for each target toxin.

\begin{tabular}{llll}
\hline & DA & OA & STX \\
\hline LOD (ng/mL) & 0.06 & 0.07 & 0.05 \\
$50 \% \mathrm{~B} / \mathrm{B}_{0}(\mathrm{ng} / \mathrm{mL})$ & 0.80 & 5.0 & 0.4 \\
WR $(\mathrm{ng} / \mathrm{mL})$ & $0.1-2.0^{*}$ & $0.1-5.0^{*}$ & $0.1-0.4^{*}$ \\
\hline
\end{tabular}

* Concentrations higher than 2.0 (for DA), 5.0 (for OA) and 0.4 (for STX) ng/ $\mathrm{mL}$ were not tested: they represent the maximum concentration levels of the standard solutions ready to use. 
discriminate between close concentrations (i.e. 0.1 and $0.2 \mathrm{ng} / \mathrm{mL}$ ).

To respect the deadlines of the SMS project, the prototype was directly tested on real marine water samples collected in Alonnisos.

\subsection{Analysis of real marine water samples collected in Alonnisos}

Analysis of DA, OA and STX in real samples of pelagic and coastal marine water, sampled in Alonnisos (see section 2.3.3) were carried out in laboratory using ELIMC assays, ELISA Kits and ASMAT, for a comparative evaluation. No detectable concentrations of OA and STX were found, while concentration levels of DA higher than the LODs of the three methods, and just below the minimum value $(0.1 \mathrm{ng} / \mathrm{mL})$ of the working ranges, were detected in both pelagic and coastal water.

\subsection{Field tests of the ASMAT on a floating-platform}

On-line suitability of ASMAT was demonstrated by a field installation within a floating platform, prepared by Systea in the port of La Spezia (Italy). After a general testing cycle, performed for checking the instrument, a full cycle of algal toxins analysis (calibration and seawater sample analysis) was executed. The results indicate the absence of the target toxins in natural samples. To date the prototype has been designed to analyze one toxin at a time, so that it was possible to carry out three calibration curves and the analysis of three samples within $24 \mathrm{~h}$. After few days of correct functioning, the reagents started showing some signs of deterioration. Therefore, the platform was dismounted and the modules were brought back to Systea laboratories where the staff is working to evaluate the performances of the refrigerated compartment (incorporated into the instrument), in order to ensure the stability of the reagents.

\section{Conclusions}

Marine biotoxins released as a consequence of toxic algal blooms represent a serious concern for public health. Current offline methods to detect marine biotoxins in seawater are limited and being based on strategy including sampling on site, transport to the laboratory and manual analysis, provide only temporary and discontinuous monitoring of coastal areas under control.

In this work the re-modeling of ELISA kits to set-up direct competitive ELIMC assays for the detection of domoic acid, okadaic acid and saxitoxin in seawater, is presented. The proposed ELIMC assays combine the high performing and stable reagent solutions, supplied by commercial ELISA kits, the convenience of a separation step through the use of magnetic beads, and the straightforwardness of the colorimetric detection. All these features match well with the challenge to develop an efficient system for in situ continuous monitoring of algal toxins in seawater. With these assumptions, after verifying the capability of the ELIMC assays to detect DA, OA and STX in seawater, full automation of the single manual operations has been implemented in the ASMAT prototype. The instrument was conceived to automatically collect seawater samples, dilute the reagents, analyze standards/samples, wash the circuit, and control/process data. At first, this novel analytical tool was tested in laboratory to assess the analytical parameters and found capable of detecting sub-ppb levels of the target toxins.

The suitability of the ASMAT for on-line monitoring of marine toxins was then demonstrated, installing the instrument (integrated with a communication module for real time data transfer to a control center) within a floating platform. Besides the promising preliminary results, there is still room for improvements in terms of stability over time.

\section{Acknowledgement}

This work was funded by the EU FP7-OCEAN-2013.1 SMS (Sensing toxicants in Marine waters makes Sense using biosensors) project under grant agreement No 613844.

\section{Appendix A. Supplementary data}

Supplementary material related to this article can be found, in the online version, at doi:https://doi.org/10.1016/j.snb.2018.12.083.

\section{References}

[1] https://coastalscience.noaa.gov/research/habs/.

[2] D.M. Anderson, Approaches to monitoring, control and management of harmful algal blooms (HABs), Ocean Coast. Manage. 52 (2009) 342-347.

[3] K.G. Sellner, G.J. Doucette, G.J. Kirkpatrick, Harmful algal blooms: causes, impacts and detection, J. Ind. Microbiol. Biotechnol. 30 (2003) 383-406.

[4] P. Visciano, M. Schirone, M. Berti, A. Milandri, R. Tofalo, G. Suzzi, Marine biotoxins: occurrence, toxicity, regulatory limits and reference methods, Front. Microbiol. 7 (2016) 1051-1061.

[5] R. Poletti, A. Milandri, M. Pompei, Algal biotoxins of marine origin: new indications from the European Union, Vet. Res. Commun. 27 (2003) 173-182.

[6] P. Hess, B. Grune, D.B. Anderson, T. Aune, L.M. Botana, P. Caricato, et al., Three rs approaches in marine biotoxin testing, ATLA 34 (2006) 193-224.

[7] A.M. Botana, P. Otero, P. Rodriguez, A. Alfonso, L.M. Botana, Current situation on analysis of marine toxins, Rev. Anal. Chem. 32 (1) (2013) 15-34.

[8] N.G. Montoia, V.K. Fulko, M.O. Carignan, J.I. Carreto, Toxin variability in cultured and natural population of Alexandrium tamarense from southern South Americaevidences of diversity and environmental regulation, Toxicon 56 (2010) $1408-1418$.

[9] I.O.M. Chan, V.W.H. Tsang, K.K. Chu, S.K. Leung, M.H.W. Lam, T.C. Lau, et al., Solid-phase extraction-fluorimetric high performance liquid chromatographic determination of domoic acid in natural seawater mediated by an amorphous titania sorbent, Anal. Chim. Acta 583 (2007) 111-117.

[10] R. Pocklington, J.E. Milley, S.S. Bates, C.J. Bird, A.S.W. de Freitas, M.A. Quilliam, Trace determination of domoic acid in sea water and phytoplankton by high-performance liquid chromatography of the fluorenylmethoxycarbonyl (FMOC) derivative, Int. J. Environ. Anal. Chem. 38 (1990) 351-368.

[11] I. Bravo, M. Fernandez, I. Ramilo, A. Martinez, Toxin composition of the toxic Dinophlagellate Prorocentrum lima isolated from different locations along the Galician coast (NW Spain), Toxicon 39 (2001) 1537-1545.

[12] H. He, H. Li, J. Yiang, F. Chen, Determination of paralithic shellfish poisoning toxins in cultured microalgae by high performance liquid chromatography with fluorescence detection, Anal. Bioanal. Chem. 383 (2005) 1014-1017.

[13] L.E. Vera-Avila, D.J. Marin-Perez, R. Covarrubias-Herrera, Trace level determination of domoic acid in seawater by off-line/on-line solid-phase extraction coupled to HPLC-UV, J. Mex. Chem. Soc. 55 (2011) 65-71.

[14] Z. Wang, K.L. King, J.S. Ramsdell, G.J. Doucette, Determination of domoic acid in seawater and phytoplankton by liquid chromatography-tandem mass spectrometry, J. Chromatogr. 1163 (2007) 169-176.

[15] M. Halme, M. Rapinoja, M. Karjalainen, P. Vanninen, Verification and quantification of saxitoxin from algal samples using fast and validated hydrophilic interaction liquid chromatography-tandem mass spectrometry method, J. Chromatogr. B-Anal Technol. Biomed. Life Sci 880 (2012) 50-57.

[16] L.L. Mafra Jr, C. Leger, S.S. Bates, M.A. Quilliam, Analysis of trace levels of domoic acid in seawater and plankton by liquid chromatrography without derivatization, using UV or mass spectrometry detection, J. Chromatogr. 1216 (2009) 6003-6011.

[17] J. Blanco, F. Livramento, I.M. Rangel, Amnesic shellfish poisoning (ASP) toxins in plankton and molluscs from Luanda Bay, Angola, Toxicon 55 (2010) 541-546.

[18] P. de la Iglesia, G. Gimenez, J. Diogene, Determination of dissolved domoic acid in seawater with reversed-phase extraction disks and rapid resolution liquid chromatography tandem mass spectrometry with head-column trapping, J. Chromatogr. 1215 (2008) 116-124.

[19] K.A. Lefebvre, B.D. Bill, a. Erickson, K.A. Baugh, L. O'Rourke, P.R. Costa, S. Nance, V. Trainer, Characterization of intracellular and extracellular saxitoxin levels in both field and cultured Alexexandrium spp. Samples from Sequim Bay, Washington, Mar. Drugs 6 (2008) 103-116.

[20] R. Del Rio, S. Bargu, D. Baltz, S. Fire, G. deterso, Z. Wang, Gulf menhaden (Brevoortia patronus): a potential vector of domoic acid in coastal Louisiana food webs, Harmful Algae 10 (2010) 19-29.

[21] S.E. McNamee, C.T. Elliott, P. Delahaut, K. Campbell, Multiplex biotoxin surface plasmon resonance method for marine biotoxins in algal and seawater samples, Environ. Sci. Pollut. Res. 20 (2013) 6794-6807.

[22] R. Congestri, L. Micheli, G. Palleschi, Monitoring domoic acid in marine phytoplankton by disposable immunosensors, Am. J. Plant Sci. 8 (2017) 1077-1091.

[23] I. Garthwaite, K.M. Ross, C.O. Miles, R.P. Hansen, D. Foster, A.L. Wilkins, N.R. Towers, Polyclonal antibodies to domoic acid, and their use in immunoassays for domoic acid in sea water and shellfish, Nat. Toxins 6 (1998) 93-104.

[24] R.W. Litaker, T.N. Stewart, B.L. Eberhart, J.C. Wekell, V.L. Trainer, R.M. Kudela, et al., Rapid enzyme-linked immunosorbent assay for detection of the algal toxin domoic acid, J. Shellfish Res. 27 (2008) 1301-1310.

[25] S. Leonardo, A. Toldrà, M. Rambla-Alegre, M. Fernández-Tejedor, K.B. Andree, L. Ferreres, Self-assembled monolayer-based immunoassays for okadaic acid detection in seawater as monitoring tools, Mar. Environ. Res. 133 (2018) 6-14.

[26] J.P. Meneely, K. Campbell, C. Greef, M.J. Lochhead, C.T. Elliott, Development and 
validation of an ultrasensitive fluorescence planar waveguide biosensor for the detection of paralytic shellfish toxins in marine algae, Biosens. Bioelectron. 41 (2013) 691-697.

[27] S.E. McNamee, C.T. Elliott, B. Greer, M. Lochhead, K. Campbell, Development of a planar waveguide microarray for the monitoring and early detection of five harmful algal toxins in water and cultures, Environ. Sci. Technol. 48 (2014) 13340-13349.

[28] G. Volpe, U. Sozzo, S. Piermarini, E. Delibato, G. Palleschi, D. Moscone, Towards the development of a single-step immunosensor based on an electrochemical screen-printed electrode strip coupled with immunomagnetic beads, Anal. Bioanal. Chem. 405 (2013) 655-663.

[29] S. Piermarini, G. Volpe, L. Micheli, D. Moscone, G. Volpe, An ELIME-array for the detection of aflatoxin B1 in corn samples, Food Control 20 (2008) 371-375.

[30] D. Romanazzo, F. Ricci, G. Volpe, C.T. Elliot, S. Vesco, K. Kroeger, et al., Development of a recombinant Fab-fragment based electrochemical immunosensor for deoxynivalenol detection in food samples, Biosens. Bioelectron. 25 (2010) 2615-2621.

[31] G. Adornetto, L. Fabiani, G. Volpe, A. De Stefano, S. Martini, R. Nenna, et al., An electrochemical immunoassay for the screening of celiac disease in saliva samples, Anal. Bioanal. Chem. 407 (2015) 7189-7196.

[32] A. De Stefano, G. Volpe, G. Adornetto, S. Bernardini, M. Nuccetelli, G. Gallucci, L. Di Ruvo, D. Moscone, Development of a very sensitive ELIME assay for detection of sIgE to G5 and D2 aeroallergens in serum samples, Electroanal 26 (2014) $1382-1389$

[33] G. Volpe, E. Delibato, L. Fabiani, E. Pucci, S. Piermarini, A. D’Angelo, F. Capuano, D. De Medici, G. Palleschi, Development and evaluation of an ELIME assay to reveal the presence of Salmonella in irrigation water:comparison with Real-Time PCR and the Standard Cultural Method, Talanta 149 (2016) 202-210.

[34] S. Bodini, L. Sanfilippo, E. Savino, P. Moscetta, Automated micro loop flow reactor technology to measure nutrients in coastal water: State of the art and field application, Proceedings of Oceans'15 MTS/IEEE Conference (2015).

Konstantinos Petropoulos received the MSc degree in Molecular Diagnostics and Biotechnology at the University of Camerino (Italy) and the PhD degree in Chemistry at University of Rome Tor Vergata. His work focused on the development and application of electrochemical biosensors/immunosensors and colorimetric methods based on the use of bio-recognition elements for target molecules of environmental and clinical interest.

Sergio Bodini received the MSc degree in Chemistry at University of Milano in 1995. After, he worked for 14 ys as a Senior researcher for a private Institute, ISRIM. Basic studies aiming at investigating bacterial stress responses, found a practical application in a EU project for the design and realization of an on-line fully automated $E$. coli analyzer, which was awarded by the European Commission as "best LIFE Environment projects 2010". Since 2011, he is Scientific Coordinator for the company Systea SpA. and he specialized in the automation of analytical chemistry and acute toxicity methods.

Laura Fabiani received the MSc degree in industrial Biotechnologies at the University of Rome Tor Vergata. Currently, she is a PhD Student of Analytical Chemistry at University of Rome Tor Vergata. She is working on the development and application of electrochemical and colorimetric immunosensors for environmental and food analysis.

Laura Micheli is Associate professor at University of Rome Tor Vergata, since 2014. The research activity is focalized on the development of disposable electrochemical tools based on bio/immunosensor technology for the determination of several analyte in food, clinical and cultural heritage fields. Great attention is devoted to their validation through the use of spectrophotometric and chromatographic methods.
Alessandro Porchetta received the PhD degree in Chemistry at University of Rome Tor Vergata. He is a researcher at the Chemistry Department of the University of Rome, Tor Vergata. His research interests lie in the area of DNA nanotechnology, with a focus on DNA-based switching biomolecules for biosensing applications in environmental and clinical analysis.

Silvia Piermarini received the MSc degree in Biology Science and the PhD degree in Chemistry at University of Rome Tor Vergata. Currently she has a permanent position at the Chemistry Department in Tor Vergata University, working on the development and application of disposable electrochemical biosensors for environmental and food analysis.

Giulia Volpe received the MSc degree in Biology Science at University of Rome La Sapienza. From 2001 she has a permanent position at the Chemistry Department in Tor Vergata University, working on the development of new sensor/biosensor systems for chemical and microbiological contaminants in food, clinical and environmental samples.

Fabio Massimo Pasquazzi received the bachelor's degree in Electronic Engineering at University of Bologna in 2006 and the MSc degree at Queen Mary University in London. Since 2011, he works for Systea SpA as a product specialist for multiparameter probes for nutrient analysis which were awarded by ACT Nutrients as best US PO4 and NO3 in situ analyzers.

Luca Sanfilippo received the MSc degree in Nuclear Engineering. He gained 30 years of experience as an environmental monitoring consultant, specialized in the management of development projects and implementation of instrumentation for the measurement of environmental parameters (air and water quality). He was invited several times by the European Commission as an evaluator of proposals for European research and metrology projects. He is an Italian member of the CEN TC230 WG4 working group "Performance Requirements for Water Monitoring Equipment".

Pompeo Moscetta received the Diploma of Industrial Electronic Engineering in 1974. From 1975-1979 he was employed at VIDEOCOLOR SpA becoming an expert in Intel 8080 microprocessor programming for industrial automation. From 1975-1979 he was employed in the Italian branch of the company Technicon INSTRUMENTS Technical Manager of the hospital Computer Division; in this period has coordinated the development of Laboratory Information Systems of about 50 of the largest Italian hospitals. In 1988 he founded Systea SpA, a company specialized in automation solutions for water chemical analysis, which still controls as Managing Director and Head of R\&D department.

Salvatore Chiavarini received the MSc degree in Chemistry and $\mathrm{PhD}$ in Chemical Sciences at University of Rome La Sapienza. Since 1988, he is working at ENEA as a Senior Researcher in the Environmental Department. His main interests are on the development of analytical protocols for environmental characterization using mass spectroscopy hyphenated techniques.

Giuseppe Palleschi received the MSc degree in Chemistry at University of Rome La Sapienza. From 1994 he is full professor of Analytical Chemistry at the Chemistry Department of the Tor Vergata University. He has been focused in the last 40 years on the development of electrochemical sensors bio- and immunosensors in the areas of environment, biomedicine, and food analysis. In the 2000 he obtained the Laurea Honoris Causa from the University of Bucharest for his activity in the area of chemical sensors for environment and food control. 